\title{
Model of coarsening and vortex formation in vibrated granular rods
}

\author{
Igor S. Aranson ${ }^{1}$ and Lev S. Tsimring ${ }^{2}$ \\ 1 Argonne National Laboratory, 9700 South Cass Avenue, Argonne, IL 60439 \\ 2 Institute for Nonlinear Science, University of California, San Diego, La Jolla, CA 92093-0402
}

(November 12, 2018)

\begin{abstract}
Neicu and Kudrolli observed experimentally spontaneous formation of the long-range orientational order and large-scale vortices in a system of vibrated macroscopic rods. We propose a phenomenological theory of this phenomenon, based on a coupled system of equations for local rods density and tilt. The density evolution is described by modified Cahn-Hilliard equation, while the tilt is described by the Ginzburg-Landau type equation. Our analysis shows that, in accordance to the Cahn-Hilliard dynamics, the islands of the ordered phase appear spontaneously and grow due to coarsening. The generic vortex solutions of the Ginzburg-Landau equation for the tilt correspond to the vortical motion of the rods around the cores which are located near the centers of the islands.
\end{abstract}

PACS: 45.70.-n, 45.70.Ht, 45.70.Qj, 83.70.Fn

Vibrated granular materials exhibit many interesting phenomena, including formation of cellular and localized patterns, convection, phase separation etc. 11 6]. Recently, Neicu and Kudrolli [7] studied the dynamics of a layer of long cylindrical grains (rods) subjected to vertical vibration, and discovered a surprising phenomenon of spontaneous formation of islands of vertically aligned rods which co-exit with the "sea" of randomly packed almost horizontal rods. Subsequently, small islands merge and form large islands which typically exhibit collective vortical motion of rods. Near the core of the vortex this motion has a form of solid body rotation, while farther away from the core, the angular velocity decays.

While the bistability and first order phase transitions leading to phase separation and coarsening are typical in granular dynamics (observed, for example, in monolayer excitation [2,3, vibrated powder heaping [5,6], electrostatically-driven granular systems [\$]) and usually caused by the inelasticity of grains, the emergence of the vortical motion within the ordered phase is rather unexpected. Experiment [7] shows that rods within a vortex are tilted in the azimuthal direction, and slowly drift in the direction of the tilt. Authors [7] suggested that the drift occurs due to the confinement of the rod vibration by its tilted neighbors.

In this Letter we introduce a continuous phenomenological model of the transition to the ordered vortical state based on the modified Cahn-Hilliard equation governing the dynamics of local rods density and the Ginzburg-Landau type equation for the tilt. Our model reproduces qualitatively the observed phase separation, coarsening and vortex formation. We derive the solutions for the stationary vortices and discuss their stability.

Model. The motion of rods is described by the momentum conservation equation in the form

$$
\rho\left(\frac{D \mathbf{v}}{D t}+\zeta \mathbf{v}\right)=-\nabla p+\alpha \mathbf{n} f_{0}(n) \rho
$$

Here $\mathbf{v}=\left(v_{x}, v_{y}\right)$ is the horizontal velocity of rods, $\rho$ is the density, $p$ is the hydrodynamic pressure, the tilt vector $\mathbf{n}=\left(n_{x}, n_{y}\right)$ is the projection of the rod on $x-y$ plane, and $n=|\mathbf{n}|$. The term $\alpha \mathbf{n} f_{0}(n) \rho$, accounts for the driving force from the vibrating bottom exerted on the tilted rod. According to experiments [7], the driving force is proportional to the tilt of rods for small tilt values, but the saturates and eventually decays to zero at $n>n_{0}$. Finally, the term $\zeta \mathbf{v}$ describes the momentum dissipation due to bottom friction. Eq. (11) must be augmented by the mass conservation equation in the from

$$
\partial_{t} \rho+\operatorname{div}(\mathbf{v} \rho)=0
$$

In the following we assume that the friction is strong, and neglect the inertia term $\frac{D \mathbf{v}}{D t}$ with respect to the friction term $\zeta \mathbf{v}$. Thus, we can express the velocity in the form

$$
\mathbf{v}=-\frac{1}{\zeta \rho}\left(\nabla p-\alpha \mathbf{n} f_{0}(n) \rho\right)
$$

Now, substituting the velocity (3) into the mass conservation law Eq. (2), we obtain

$$
\partial_{t} \rho=\zeta^{-1} \operatorname{div}\left(\nabla p-\alpha \mathbf{n} f_{0}(n) \rho\right)
$$

To describe phenomenologically the experimentally observed phase separation and coarsening we employ the Cahn-Hilliard approach (see for review 10]). We assume that the pressure $p$ can be obtained from the variation of certain "free energy" type functional of the $\rho$ field

$$
p=\frac{\delta F}{\delta \rho}
$$

We adopt the standard form of the free energy taking into account the local dynamics and diffusive-type coupling

$$
F=\iint d x d y\left(l^{2}(\nabla \rho)^{2}+f(\rho)\right)
$$

where $l$ is the characteristic length scale related to the rod thickness. To account for the bistability and phase separation, function $f$ should have two minima separated by a 
maximum. For simplicity we choose a quartic polynomial form of $f$, and define $d f / d \rho=A\left(\rho-\rho_{0}\right)\left(\rho_{*}-\rho\right)(\delta-\rho)$, where $0 \leq \rho_{0}<\delta<\rho_{*}$ are characteristic rod densities depending on the driving acceleration 11]. Substituting (5) and (6) into Eq. (4), after appropriate rescaling we obtain the modified Cahn-Hilliard equation

$$
\begin{aligned}
\partial_{t} \rho & =-\nabla^{2}\left(\nabla^{2} \rho-\rho(1-\rho)(\delta-\rho)\right) \\
& -\alpha \operatorname{div}\left(\mathbf{n} f_{0}(n)\left(\rho+\rho_{0}\right)\right),
\end{aligned}
$$

where we keep the same notations for the rescaled variables and parameters.

To close the description we need to add an equation for the evolution of tilt $\mathbf{n}$. For density $\rho<1$ the vertical orientation of rods corresponding to $\mathbf{n}=0$ is unstable, as rods spontaneously tilt. We assume that the growth rate of the instability depends on the rods packing density, so we can write for the local dynamics

$$
\partial_{t} \mathbf{n}=f_{1}(\rho) \mathbf{n}-|\mathbf{n}|^{2} \mathbf{n}
$$

with $f_{1}(\rho)=a_{0}-a_{1} \rho, a_{0,1}>0$ some constants. As $\rho$ increases, the instability saturates and the equilibrium tilt diminishes. In addition, rods interact with each other, which leads to the additional spatial derivative operator $\hat{D}[\mathbf{n}]$ in Eq.(8). Since the tilt field is not divergencefree, from the general symmetry considerations, in the lowest (second) order, the "diffusion" operator acting on $\mathbf{n}$, takes the form $\hat{D}[\mathbf{n}]=f_{2}(\rho)\left(\xi_{1} \nabla^{2} \mathbf{n}+\xi_{2} \nabla \operatorname{div} \mathbf{n}\right)$. The coefficients $\xi_{1,2}$ in this expression are analogous to the first and second viscosity in ordinary fluids (see [9]). Function $f_{2}(\rho)$ describes the decrease of the spatial coupling strength as the rods density decreases. We assume that in the gas phase $(\rho \rightarrow 0)$ the spatial coupling between the rods is small and their tilt becomes large and uncorrelated. Accordingly, we set $f_{2}=\rho$, if $\rho>0$ and $f_{2}=0$ otherwise. Finally, we include the simplest term describing coupling between the tilt and the density gradient $\beta \nabla \rho$. Combining all these terms we arrive at

$$
\begin{aligned}
\partial_{t} \mathbf{n} & =f_{1}(\rho) \mathbf{n}-|\mathbf{n}|^{2} \mathbf{n}+ \\
& +f_{2}(\rho)\left(\xi_{1} \nabla^{2} \mathbf{n}+\xi_{2} \nabla \operatorname{div} \mathbf{n}\right)+\beta \nabla \rho .
\end{aligned}
$$

It is convenient to introduce new complex variable $\psi=n_{x}+i n_{y}$. Then, Eq. (9) assumes the form of the generalized Ginzburg-Landau equation $\left(\bar{\xi}=\xi_{1}+\xi_{2} / 2\right)$

$$
\begin{aligned}
\partial_{t} \psi & =\left(f_{1}(\rho)-|\psi|^{2}\right) \psi+\beta\left(\partial_{x}+i \partial_{y}\right) \rho \\
& +f_{2}(\rho)\left(\bar{\xi} \nabla^{2} \psi+\frac{\xi_{2}}{2}\left(\partial_{x}+i \partial_{y}\right)^{2} \psi^{*}\right)
\end{aligned}
$$

Phase separation. Eq. (7) exhibits phase separation only in a certain range of initial conditions $\rho$ and parameter $\delta$. The total mass conservation yields $\iint \rho d x d y=$ $S \Phi$, where $S$ is the cavity area, and $\Phi$ is the average density determined by the filling fraction. Stationary solution to Eq. (7) obeys

$$
\nabla^{2} \rho-\rho(1-\rho)(\delta-\rho)=B
$$

where $B=$ const is determined below. In the phase separation regime spatially-homogeneous Eq. (11) has three real roots $\rho_{1}<\rho_{2}<\rho_{3}$. The root $\rho_{2}$ corresponds to the unstable solution and $\rho_{1,3}$ to the stable ones. Final stage of the phase separation leads to one domain of the high-density phase (solid) $\rho=\rho_{3}$ of the area $S_{h}$ and the low-density one (gas) $\rho=\rho_{1}$ of the area $S_{l}=S-S_{h}$. From the mass conservation one obtains

$$
S_{h} \rho_{3}+\left(S-S_{h}\right) \rho_{1}=S \Phi .
$$

Here we neglect the interfacial contributions assuming that $S_{l}, S_{h} \gg 1$. In addition, Eq. (12) must be augmented by the condition that the free energy densities of the both phases are equal, which is expressed by the relation (so-called area rule, see e.g. 12])

$$
\left.\int_{\rho_{1}}^{\rho_{3}}[\rho(1-\rho)(\delta-\rho)-B)\right] d \rho=0 .
$$

Eq. (13) and Eq. (11) fix the value of $B$, and, correspondingly the roots $\rho_{1,3}$. ¿From Eq. (12) it follows that the solution with two phases is possible if $\rho_{1}<\Phi<\rho_{3}$. It defines the phase separation region in Fig. 1.

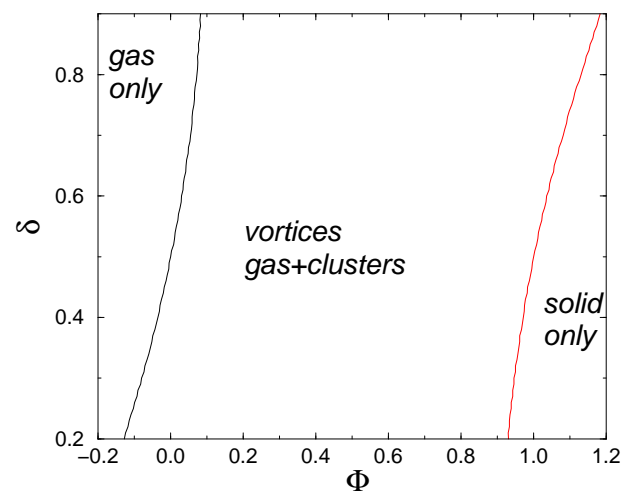

FIG. 1. Phase diagram in $\Phi-\delta$ plane. Here $\Phi$ is related to the filling fraction and $\delta$ to the driving acceleration.

Let us consider radially symmetric vortex solutions to Eq. (10). In this case $\rho$ is a function of the polar radius $r$, and $\psi$ can be expressed as $\psi=\exp ( \pm i \theta) w(r)$, where $w$ is a complex function, and $\theta$ is the polar angle (for definiteness we take sign + ). Using $\partial_{x}+i \partial_{y}=$ $\exp (i \theta)\left(\partial_{r}+i / r \partial_{\theta}\right)$, we obtain from Eq. (10)

$$
\partial_{t} w=f_{2}\left(\bar{\xi} \nabla_{r}^{2} w+\frac{\xi_{2}}{2} \nabla_{r}^{2} w^{*}\right)+f_{1} w-|w|^{2} w+\beta \rho_{r}
$$

where $\nabla_{r}^{2}=\partial_{r}^{2}+r^{-1} \partial_{r}-r^{-2}$ is the radial Laplacian operator. For $\xi_{2}, \beta=0$, Eq. (14) possesses a stationary solution in the form $w=W(r) \exp \left(i \phi_{0}\right)$ with the real positive magnitude $W$ and an arbitrary constant phase $\phi_{0}$. The terms $\propto \xi_{2}, \beta$ still permit the constant phase 
solutions, but they destroy the continuous phase degeneracy. Indeed, Eq. (14) for $\beta=0$ yields

$$
f_{2}\left(\bar{\xi}+\frac{\xi_{2}}{2} \cos 2 \phi_{0}\right) \nabla_{r}^{2} W+f_{1} W-W^{3}=0
$$

and $\sin 2 \phi_{0}=0$. Solutions exist only for $\phi_{0}=0, \pi$ or $\pm \pi / 2$. Function $W$ describes the standard (and welldocumented) vortex solution to the Ginzburg-Landau equation with the property $W \rightarrow f_{1}^{1 / 2}$ for $r \rightarrow \infty$ and $W \sim r$ for $r \ll 1$ (for its rational approximation see e.g. [13]). Solutions with $\phi_{0}=0, \pi$ describe sinks (sources) with zero circulations, whereas the solutions with $\phi_{0}= \pm \pi / 2$ are vortices with non-zero circulations. The sign of $\phi_{0}$ determines the direction of rotation. Near the vortex core $W \sim r$, which corresponds to the solid body rotation, as velocity $v_{\theta} \sim W$. Far away from the core the vortex exhibits differential rotation.

For $\beta \partial_{r} \rho \neq 0$, Eq. (14) has constant phase solutions only with $\phi_{0}=0, \pi$, i.e. with zero circulation. However, it does not guarantee the selection of this solution in the bulk of large islands where the density gradient is small. It is easy to show that solutions with $\phi_{0}=0$ are energetically unfavorable with respect to rotating solutions with $\phi_{0}= \pm \pi / 2$ if $\beta \partial_{r} \rho$ is small. Indeed, Eq. (14) for $\beta \partial_{r} \rho=0$ can be written in the form

$$
\partial_{t} w=-\frac{\delta U}{\delta w^{*}}
$$

with the free energy functional

$$
\begin{aligned}
U & =\int d \mathbf{r}\left[f_{2} \bar{\xi}\left(\left|\partial_{r} w\right|^{2}+r^{-2}|w|^{2}\right)\right. \\
& \left.+\frac{f_{2} \xi_{2}}{4}\left(\left(\left(\partial_{r}+r^{-1}\right) w^{*}\right)^{2}+\text { c.c. }\right)-f_{1}|w|^{2}+|w|^{4}\right]
\end{aligned}
$$

Substituting vortex solution $w=W(r) \exp \left(i \phi_{0}\right)$ in Eq. (17), one obtains after integration (since the calculation of the vortex energy is rather straightforward, we refer interested readers to Ref. [13], p. 11).

$$
U=f_{1} f_{2}\left(\bar{\xi}+\xi_{2} / 2 \cos 2 \phi_{0}\right) \log R / r_{0}+\text { const }
$$

where $r_{0} \sim O(1)$ has the meaning of core radius, and $R$ is the outer cutoff radius of integration. As one sees from Eq. (18), for physically realizable case $\xi_{2}>0$, the vortices with $\phi_{0}= \pm \pi / 2$ have lower energy, and therefore are more energetically favorable, and are selected in dynamics. In a general case, the vortex solution would have a radius-dependent phase $\phi_{0}$. Near the center where the density is almost constant, the phase would be close to $\pm \pi / 2$, and near the island border where the density decreases rapidly, the phase should approach 0 or $\pi$. This scenario suggests that the azimuthal velocity should grow with radius near the core, and decrease near edge of the vortex, which is confirmed by our numerical simulations (see below) and agrees with experiments. In addition, one can show that for $\beta>0$ the term $\beta \nabla \rho$ considered as a small perturbation, leads to the drift of the vortex core towards the gradient of density, and therefore it stabilizes the vortex core near the center of the island.

Numerical simulations of the Cahn-Hilliard equation (7) were performed using an FFT split-step method, and the Ginzburg-Landau equation (10) was solved using explicit method. The domain of integration was $100 \times 100$ dimensionless units with periodic boundary conditions, number of mesh points/FFT harmonics was $256 \times 256$. As initial conditions we used $\rho \approx \delta$ with with small amplitude noise and random initial conditions for $\psi$. Selected results are presented in Fig. 2-5.

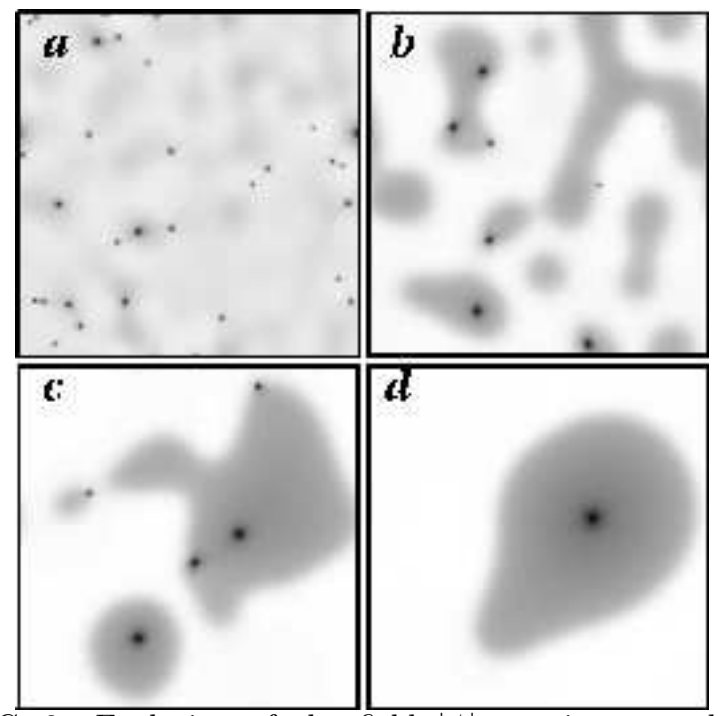

FIG. 2. Evolution of the field $|\psi|$, vortices are shown as black dots, white area corresponds to larger $|\psi|$ (and $\rho)$, dark vise versa. Parameters: $\bar{\xi}=1, \xi_{2}=1$, $\beta=0.04, f_{0}=1.5-|\psi|^{2}, f_{1}=1.4-0.7 \rho$, $\delta=0.3, \rho_{0}=0.25, \alpha=0.03$. Images are shown for $t=80(a), 400(b), 1600(c)$ and $t=3200(d)$.

At the initial stage of the evolution many vortices and small dense clusters (islands) are created throughout the domain of integration (Fig. 2a). Islands are seen as darker areas on the figure because an increase in density $\rho$ results in the decrease of the amplitude of $\psi$. Some islands trap vortices and are practically immobile, others don't contain vortices and drift in the direction defined by the mean value of the tilt $\mathbf{n}$. With time, small islands disappear and bigger islands grow (Fig. 2b,c). It is interesting to note that due to tilt-driven drift coarsening occurs much faster than in ordinary Cahn-Hilliard dynamics. Finally, one big island with the vortex in the center is formed (Fig. 2d). Corresponding density $\rho$ and the phase field $\arg \psi$ are shown in Fig. 3. Surprisingly, even far from the island there some "dormant vortices" in the low-density phase (gas). These vortices are seen as the end-points of the phase singularity lines (lines between dark and white) in Fig. 3b. These vortices do not 
annihilate because in the low-density phase the diffusion terms in Eq. 10 are absent. They are not seen in Fig. $3 \mathrm{c}$ because their core size is small in the gas phase. However, these vortices can be advected into the high-density phase from the edges.
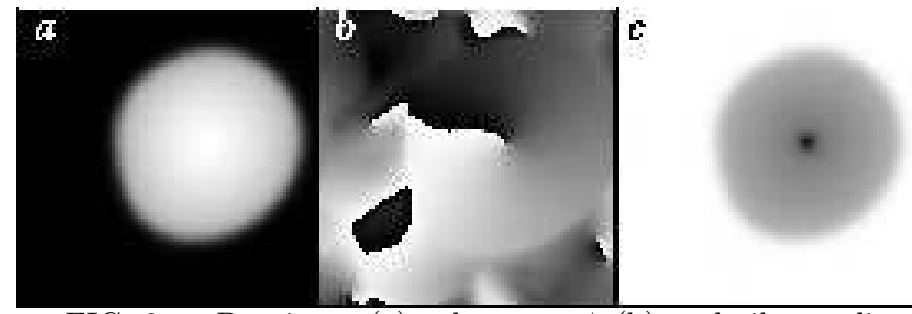

FIG. 3. Density $\rho$ (a), phase $\arg \psi$ (b) and tilt amplitude $|\psi|$ (c) for $t=4960$, other parameters as in Fig. 2 . Black corresponds to $\rho=0=\arg \psi=|\psi|=0$, white to $\rho=1.4, \arg \psi=2 \pi,|\psi|=1.2$.

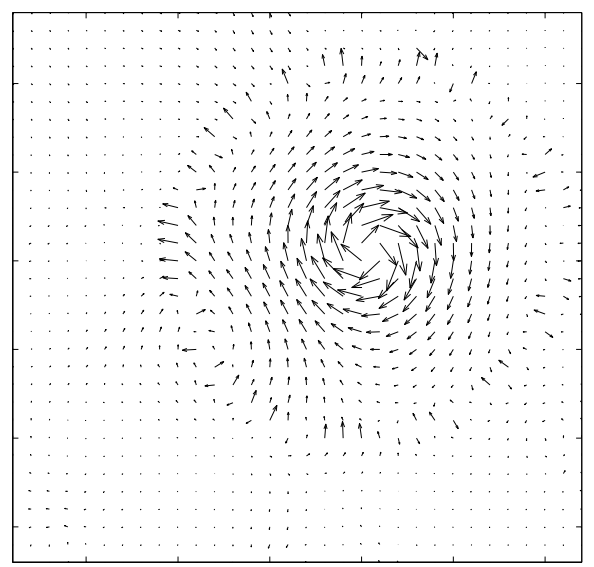

FIG. 4. Velocity field corresponding to Fig. 3.

The velocity field is calculated using Eq. (3). Fig. A shows the velocity field corresponding to the calculated tilt structure of Fig.3. The rods perform circular motion around the vortex center. The azimuthal velocity $v_{\theta}$ vs $r$ is shown in Fig. 5. The velocity is maximal somewhere between the core and the island edge. It qualitatively resembles the experimental one [7]. Outside the island the tilt becomes large and the velocity becomes small.

In conclusion, we developed a phenomenological model of the formation of the vortical ordered state in the system of vertically vibrated rods. Our continuum model is based on a Cahn-Hilliard equation for the rods aerial density coupled to the Ginzburg-Landau equation for the rod tilt. The model reproduces the qualitative features of the vortex formation process observed in the recent experiment [7]. We thank Toni Neicu and Arshad Kudrolli for stimulating discussions. This work was supported by the U.S.Department of Energy under grants W-31-109-ENG-38 and DE-FG03-95ER14516. Simulations were performed at the National Energy Research
Scientific Computing Center.

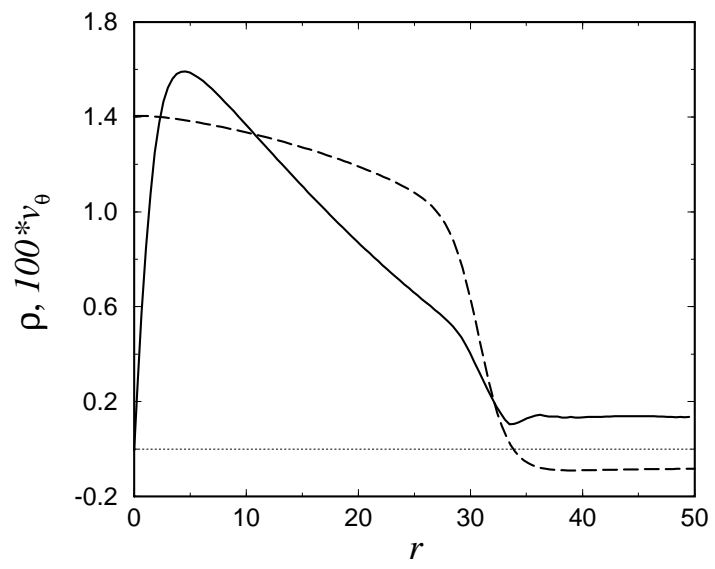

FIG. 5. Azimuthal velocity $v_{\theta}$ (solid line) and density $\rho$ (dashed line) vs $r$ for the parameter of Fig. 目.

[1] P.B. Umbanhowar, F. Melo, and H.L. Swinney, Nature (London) 382, 793-796 (1996)

[2] J.S. Olafsen and J.S. Urbach, Phys. Rev. Lett. 81, 4369 (1998)

[3] X. Nie, E. Ben-Naim, and S.Y. Chen, Europhys. Lett. 51, 679 (2000)

[4] W. Losert, D.G.W. Cooper, and J.P. Gollub, Phys. Rev. E 59, 5855 (1999)

[5] T. Shinbrot, Granular Matter 1145 (1998).

[6] J.Duran, Phys. Rev. Lett. 84, 5126 (2000); ibid 87, 254301 (2001)

[7] T.Neicu and A.Kudrolli, Vortices in vibrated granular rods, submitted to Phys. Rev. Lett. (2002).

[8] I.S. Aranson et al., Phys. Rev. Lett. 84, 3306 (2000); cond-mat/0107443

[9] L.D. Landau and E.M. Lifshits, Fluid Mechanics, Pergamon Press, New York, 1987

[10] A.J. Bray, Adv. Phys. 43, 357 (1994)

[11] Densities $\rho_{0}$ and $\rho^{*}$ in general do not coincide with the minimum and maximum equilibrium density and depend on the filling fraction $\phi$ (see Eq. (11)). Experimentally, the minimum equilibrium density corresponds to a thickness of the layer of randomly packed rods about half the rod length, and the maximum density corresponds to the densely packed vertically aligned rods. It is possible to find a bistable (non-polynomial) function $f$ with the extremal values only weakly depending on the filling fraction $\Phi$. However, it would not not change the qualitative behavior of the system.

[12] B. Meerson, Rev. Mod. Phys. 68, 215 (1996)

[13] L.M. Pismen, Vortices in Nonlinear Fields, Clarendon Press, Oxford, 290 pp, 1999. 